\title{
A epidemia de dengue/dengue hemorrágico no município do Rio de Janeiro, 2001/2002
}

\author{
The epidemic of dengue and hemorrhagic dengue fever in the city \\ of Rio de Janeiro, 2001/2002
}

\author{
Clarisse Guimarães Casali ${ }^{1}$, Marcelo Ricardo Reis Pereira ${ }^{1}$, Luciana Maria Jabor Garcia \\ Santos $^{1}$, Maíla Naves Pereira Passos ${ }^{1}$, Bruno de Paula Menezes Drumond Fortes ${ }^{1}$, Luis Iván \\ Ortiz Valencia ${ }^{2}$, Aline de Jesus Alexandre ${ }^{1}$ e Roberto de Andrade Medronho ${ }^{1,2}$
}

\begin{abstract}
RESUM0
O objetivo deste estudo foi avaliar a ocorrência dos principais sinais e sintomas dos casos de dengue clássico e dengue hemorrágico na epidemia de 2001-2002 do município do Rio de Janeiro. Foram analisados os 155.242 casos notificados ao Sistema de Informações de Agravos de Notificação, desde janeiro/2001, até junho/2002; deste total, excluindo-se os ignorados, 81.327 casos foram classificados como dengue clássico e 958 como dengue hemorrágico, com um total de 54 óbitos. Avaliaram-se as variáveis referentes à sintomatologia da doença. Manifestações gerais como febre, cefaléia, prostração, mialgia, náuseas e dor retro-orbitária tiveram alta incidência tanto no dengue clássico como no dengue hemorrágico. Por outro lado, manifestações hemorrágicas e algumas de maior gravidade como choque, hemorragia digestiva, petéquias, epistaxe, dor abdominal e derrame pleural, estiveram significativamente associadas ao dengue hemorrágico. Além disso, a evolução do quadro clínico para o óbito foi 34,8 vezes maior no dengue hemorrágico que no dengue clássico (OR=34,8; IC 19,7-61,3).
\end{abstract}

Palavras-chaves: Dengue. Epidemiologia. Sinais e sintomas. Vigilância epidemiológica.

\section{ABSTRACT}

The following study was intended to evaluate the occurrence of typical signs and symptoms in the cases of classic dengue and hemorrhagic dengue fever, during the 2001-2002 epidemic in the city of Rio de Janeiro. The authors reviewed 155,242 cases notified to the Information System of Notification Diseases, from January/2001 to June/2002: 81,327 cases were classified as classic dengue and 958 as hemorrhagic dengue fever, with a total of 60 deaths. Common symptoms, such as fever, headache, prostration, myalgia, nausea and retro-orbital pain, had a high incidence in both classic and hemorrhagic dengue fever. On the other hand, hemorrhagic signs and other signs of severe disease, such as shock, gastrointestinal bleeding, petechiae, epistaxis, abdominal pain and pleural effusion, were strongly associated to hemorrhagic dengue fever. Besides, the occurrence of death was 34.8 times higher in hemorrhagic dengue fever than in classic dengue ( $\mathrm{OR}=34.8$; CI 19.7-61.3).

Key-words: Dengue. Epidemiology. Signs and symptoms. Epidemiologic surveillance.

0 vírus do dengue pertencente ao gênero flavivírus, família flaviviridae, apresenta quatro sorotipos (1, 2, 3, 4). Fatores como a urbanização acelerada, facilidades de transporte entre regiões e falência dos programas de controle do Aedes aegypti favorecem 0 aumento da transmissão do dengue ${ }^{91011}$.
A infecção possui um espectro que varia desde a forma assintomática até quadros de hemorragia e choque, podendo evoluir, inclusive para 0 êxito letal. 0 dengue é uma doença febril aguda, com duração de 5 a 7 dias. 0 dengue clássico apresenta quadro clínico muito variável, geralmente com

\footnotetext{
1. Departamento de Medicina Preventiva da Faculdade de Medicina da Universidade Federal do Rio de Janeiro, Rio de Janeiro, RJ. 2. Núcleo de Estudos de Saúde Coletiva da Universidade Federal do Rio de Janeiro, Rio de Janeiro, RJ.

Endereço para correspondência: Dr. Roberto de Andrade Medronho. DMP/FM/UFRJ. Av. Brigadeiro Trompowsky s/nº, Cidade Universitária, $21949-900$ Rio de Janeiro, RJ. Telefax: $55212270-0097$

e-mail: medronho@nesc.ufrj.br

Recebido para publicação em 11/7/2003

Aceito em 19/5/2003
} 
febre alta $\left(39^{\circ}\right.$ a $\left.40^{\circ}\right)$ de início abrupto, seguida de cefaléia, mialgia, prostração, artralgia, anorexia, astenia, dor retro-orbitária, náuseas, vômitos e exantema. Associada à síndrome febril, em alguns casos pode ocorrer hepatomegalia dolorosa e, principalmente, nas crianças, dor abdominal generalizada. Os adultos podem apresentar manifestações hemorrágicas, como petéquias, epistaxe, gengivorragia, sangramento gastrintestinal, hematúria e metrorragia. Com o desaparecimento da febre, há regressão dos sinais e sintomas, podendo ainda persistir a fadiga ${ }^{134}$.

Por outro lado, os sintomas iniciaisdo dengue hemorrágico, apesar de semelhantes aos do dengue clássico, podem evoluir rapidamente para manifestações hemorrágicas e choque. Oscasos típicos do dengue hemorrágico são caracterizados por febre alta, fenômenos hemorrágicos, hepatomegalia e insuficiência circulatória. Nos casos graves, 0 choque ocorre entre $03^{0}$ e 70 dia de doença, geralmente precedido por dores abdominais. Sua duração é curta, podendo levar ao óbito em 12 a 24 horas ou à recuperação rápida após terapia apropriada. Um achado laboratorial importante do dengue hemorrágico é a trombocitopenia com hemoconcentração concomitante. A gravidade do dengue hemorrágico está relacionada à efusão do plasma, caracterizada por valores crescentes do hematócrito ${ }^{134}$.

Convém ressaltar que, diante da gravidade da epidemia 20012002, na qual circularam os sorotipos 1, 2 e 3, com predomínio deste último e da ameaça de reurbanização da febre amarela, transmitida pelo mesmo vetor ${ }^{5}$, torna-se necessária uma investigação aprofundada desta epidemia. Logo, um estudo descrevendo o perfil clínico e epidemiológico dos casos de dengue e dengue hemorrágico, notificados na epidemia 2001/2002 no município do Rio de Janeiro é importante para que se conheça melhor a gravidade e a magnitude do processo epidêmico.

\section{MATERIAL E MÉTODOS}

Área de estudo. 0 município do Rio de Janeiro possui uma população de 5.857.904 habitantes (IBGE, 2000). Constitui-se em um importante pólo turístico, além de político e econômico; 0 que favorece 0 aparecimento de novas doenças e a re-introdução de outras já erradicadas. 0 município possui elevado grau de desigualdade social com parte da população vivendo em condiçõos precárias, como em áreas de favelas. Além disso, o Rio de Janeiro apresenta elevada densidade demográfica e sofre um processo de urbanização desordenada.

Coleta e análise do material. Neste estudo, analisaramse 155.242 casos notificados pelo Sistema de Informações de Agravos de Notificação (SINAN), no período entre 10 de janeiro de 2001 e 22 de junho de 2002, fornecidos pela Secretaria Municipal de Saúde do Rio de Janeiro (SMS). Foram considerados apenas os casos classificados pelo SINAN como dengue clássico e dengue hemorrágico.

Análise de dados. A análise foi feita utilizando as variáveis referentes à sintomatologia, à evolução do quadro clínico (óbito), ao sexo e à idade em função do diagnóstico final, através dos programas Epi info 6.04d e o S-Plus 2000 (Release 3 Copyright 1988-2000 Math Soft, Inc). Foi utilizado 0 teste $\chi^{2}$ para diferença de proporções com nível de significância menor que $5 \%$ e cálculo do odds ratio (OR) com intervalo de confiança de $95 \%$.

\section{RESULTAD0S}

De acordo com o campo diagnóstico final (DIAGFINT) do banco de dados do SINAN foram classificados, segundo critérios da Organização Mundial de Saúde (OMS) , 81.327 casos como dengue clássico, 958 como dengue hemorrágico, 1.448 casos descartados, 3.202 casos inconclusivos, 38.163 casos ignorados, além de 30.144 notificações sem nenhuma informação referente ao campo diagnóstico final. No campo evolução ( EVOLUÇÃO) existia um total de 60 óbitos, dos quais 23 ocorreram no casos classificados como dengue clássico, 31 nos casos de dengue hemorrágico, além de 6 óbitos entre os casos descartados.

A Figura 1 mostra que a sintomatologia mais freqüente nos casos de dengue clássico na epidemia de 2001/2002 no município do Rio de Janeiro foi: febre $(98,8 \%)$, cefaléia $(95,8 \%)$, mialgia $(92,4 \%)$, prostração $(90 \%)$, dor retroorbitária $(82,5 \%)$, artralgia $(76,4 \%)$ e náusea $(70,8 \%)$.

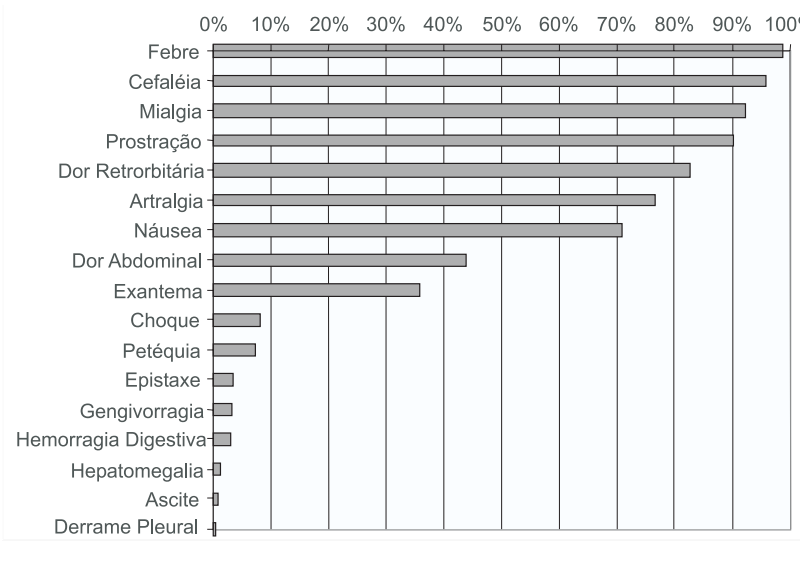

Fonte: SINAN, Secretaria Municipal de Saúde - RJ

Figura 1 - Sintomatologia do dengue clássico na epidemia de 2001/ 2002, Município do Rio de Janeiro.

A Figura 2 apresenta a sintomatologia dos casos de dengue hemorrágico. Os sinais e sintomas mais freqüentes são febre (99,2\%), cefaléia (94,7\%), mialgia (94,4\%), prostração (91,9\%), dor retro-orbitária (83,7\%), náusea (81,9\%) e artralgia (76\%).

É importante ressaltar que as manifestações hemorrágicas e de maior gravidade como dor abdominal $(55,4 \%)$, petéquias $(51,6 \%)$, hemorragia digestiva $(37,6 \%)$, gengivorragia $(34,6 \%)$, epistaxe $(34,1 \%)$, choque $(15,3 \%)$, hepatomegalia $(6,4 \%)$, ascite $(3,1 \%)$ e derrame pleural $(3,1 \%)$ estiveram mais presentes nos casos de dengue hemorrágico.

Avaliou-se também a chance de um indivíduo com dengue hemorrágico apresentar determinada sintomatologia em relação ao dengue clássico ( Tabela 1). Constatou-se que não houve 


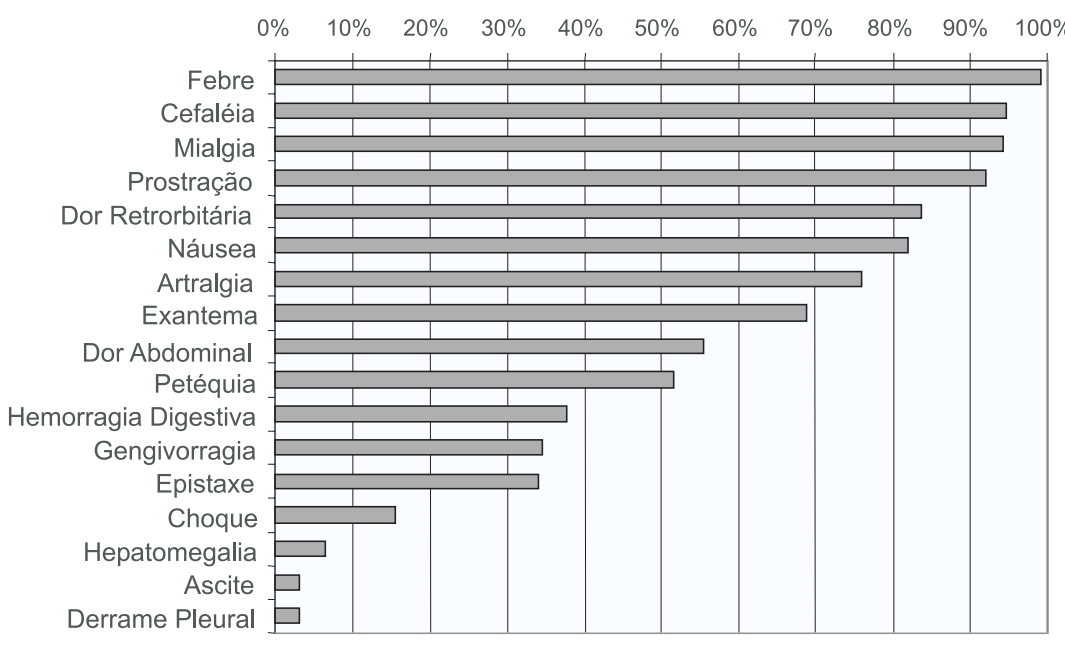

Fonte: SINAN, Secretaria Municipal de Saúde - RJ.

Figura 2 - Sintomatologia do dengue hemorrágico na epidemia de 2001/2002, Município do Rio de Janeiro.

diferenças significativas entre as manifestações gerais como: febre, cefaléia, dor retro-orbitária, mialgia, prostração e artralgia entre as duas apresentações da doença. Por outro lado, manifestações hemorrágicas e outras indicadoras de maior gravidade como náusea, dor abdominal, petéquias, epistaxe, gengivorragia, hepatomegalia, hemorragia digestiva, choque, ascite e derrame pleural estiveram mais presentes nos casos de dengue hemorrágico. Além disso, a evolução do quadro clínico para 0 óbito foi 34,75 vezes maior no dengue hemorrágico que no dengue clássico.

A média das idades tanto nos casos de dengue clássico como nos de dengue hemorrágico foi de 32,8 anos. Não houve diferença significativa entre os sexos.

A Figura 3 apresenta a evolução das principais epidemias de dengue ocorridas no município do Rio de Janeiro, sendo elas: 1986/1987 (introdução do sorotipo 1 do vírus); 1990/1991 (introdução e predominância do sorotipo 2); 2001/2002 (introdução e predominância do sorotipo 3). Constatou-se que, em um primeiro momento, logo após a entrada de um novo

Tabela 1 - Risco de um indivíduo com dengue hemorrágico apresentar um determinado sinal ou sintoma em função do dengue clássico na epidemia 2001/2002, Município do Rio de Janeiro.

\begin{tabular}{lrrr}
\hline Sintomatologia & OR & IC $(95 \%)$ & $\mathrm{p}$-valor \\
\hline Mialgia & 1,39 & $1,03-1,87$ & 0,03 \\
Náusea & 1,86 & $1,53-2,26$ & 0,00 \\
Exantema & 3,96 & $3,35-4,69$ & 0,00 \\
Dor abdominal & 1,59 & $1,35-1,87$ & 0,00 \\
Petéquia & 13,89 & $11,80-16,36$ & 0,00 \\
Epistaxe & 14,89 & $12,44-17,83$ & 0,00 \\
Gengivorragia & 15,80 & $13,18-18,92$ & 0,00 \\
Hepatomegalia & 6,90 & $4,58-10,41$ & 0,00 \\
Hemorragia digestiva & 19,73 & $16,53-23,55$ & 0,00 \\
Choque & 2,05 & $1,59-2,63$ & 0,00 \\
Ascite & 4,36 & $2,48-7,67$ & 0,00 \\
Derrame pleural & 7,64 & $4,31-13,54$ & 0,00 \\
Óbitos & 34,75 & $19,69-61,30$ & 0,00 \\
\hline
\end{tabular}

Fonte: SINAN, Secretaria Municipal de Saúde - RJ. sorotipo, ocorreu um aumento da incidência da doença, em uma primeira fase de cada período epidêmico. Seguiu-se um período em que o número de casos reduziu-se drasticamente. Após este período, ocorreu um grande aumento no número de casos, coincidindo, aproximadamente, com 0 verão.

$1986 / 1987$

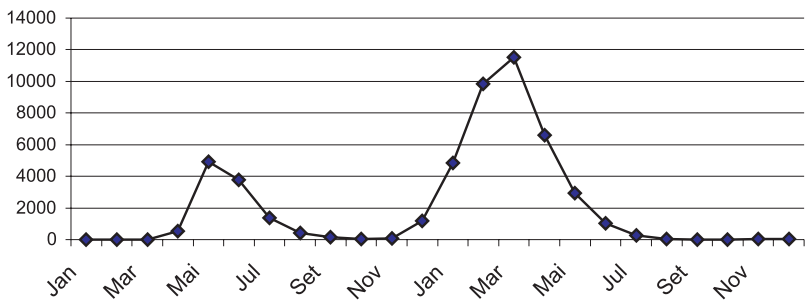

$1990 / 1991$

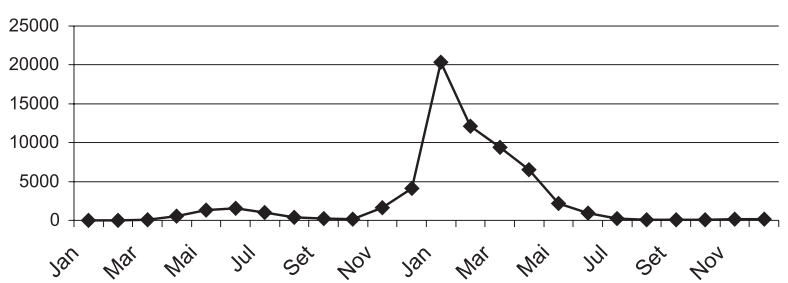

$2001 / 2002$

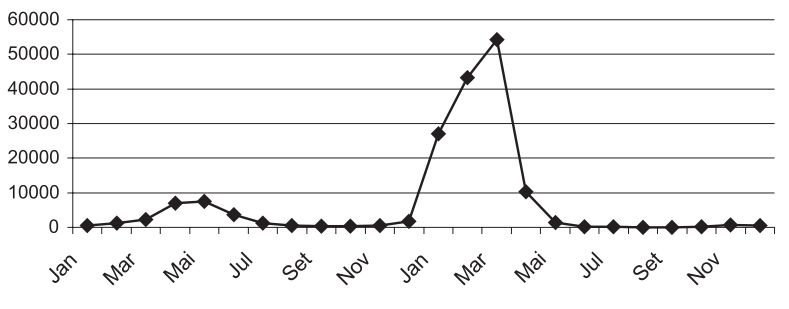

Fonte: Secretaria Municipal de Saúde - RJ

Figura 3 - Séries históricas de três epidemias de dengue no município do Rio de Janeiro. 


\section{DISCUSSÃ0}

0 município do Rio de Janeiro vem sofrendo sucessivas epidemias de dengue desde 1986, predominantemente no período de verão. Em 2001/2002 ocorreu a maior e mais grave delas.

As manifestações gerais da doença tiveram alta ocorrência tanto no dengue clássico como no dengue hemorrágico, não havendo diferença estatisticamente significativa. Isso pode ser justificado pela característica fisiopatológica do processo infeccioso, onde tais manifestações fazem parte do quadro clínico inicial, constituindo-se, inclusive, em critério diagnóstico para a doença. Por outro lado, manifestações hemorrágicas e, algumas indicativas de maior gravidade, como náusea, dor abdominal, petéquias, epistaxe, gengivorragia, hepatomegalia, hemorragia digestiva, choque, ascite e derrame pleural estiveram mais presentes nos casos de dengue hemorrágico. Além disso, verificou-se que a evolução para 0 óbito foi significativamente maior no dengue hemorrágico.

Nos três períodos epidêmicos analisados ocorreu a introdução de um novo sorotipo do vírus no município, constatando-se um comportamento muito semelhante na dinâmica do processo infeccioso na população afetada. Logo após a introdução do sorotipo, ocorre um aumento do número de casos em função da densidade vetorial e da população suscetível. Em seguida, verifica-se uma drástica queda do número de casos, coincidente com o período de meses frios, entretanto, sem que 0 vírus deixe de circular na população. Com a chegada dos meses quentes, há uma elevação da temperatura, dos índices pluviométricos e da umidade relativa do ar, fatores ambientais altamente propícios à proliferação do vetor da doença. Assim, uma nova onda epidêmica ocorre, desta vez, assumindo um caráter explosivo. Este fato possivelmente está relacionado ao controle ineficiente do vetor da doença, tendo o comportamento do processo epidêmico sido regulado fundamentalmente por questões de ordem climática e populacional. Destaca-se também que, ao se comparar a incidência do dengue na epidemia de 2001/2002, com as demais epidemias, é possível aferir que esta apresentou maior magnitude.

0 elevado número de casos de dengue hemorrágico e de óbitos é, possivelmente, justificado pelo predomínio de casos do sorotipo 3 na epidemia de 2001/2002, enquanto em epidemias passadas predominaram os sorotipos 1 e $2^{678}$. Tal fato se relaciona à introdução de um novo sorotipo em uma determinada região, onde a população já foi previamente exposta à infecção por outros sorotipos do dengue, possuindo, entretanto, susceptibilidade ao novo sorotipo. Neste caso, pode ocorrer uma resposta imunológica exacerbada à segunda infecção, podendo resultar em uma forma mais grave da doença. Além disso, a gravidade desta epidemia pode estar relacionada à introdução de uma cepa do sorotipo 3 com virulência elevada em uma região com alta densidade vetorial. Autores cubanos têm proposto uma hipótese integral de multicausalidade, onde a interação de fatores de risco individuais, virais e epidemiológicos promoveria condições para a ocorrência do dengue hemorrágico ${ }^{4}$.

Por fim, énecessário ressaltar possíveis viéses nos resultados. A provável subnotificação de casos poderia acarretar um viés de seleção. Além disso, a despeito de existir um instrumento padronizado de coleta de dados não se pode assegurar que 0 mesmo foi preenchido de maneira homogênea. Tal fato pode ocasionar erros na aferição das variáveis em estudo levando a uma distorção nas estimativas de efeito, caracterizando-se assim um possível viés de informaçã $0^{12}$.

\section{AGRADECIMENTOS}

Às Dras. Meri Baran e Cecília Nicolai da Secretaria Municipal de Saúde do Rio de Janeiro pelo fornecimento dos dados.

\section{REFERÊNCIAS BIBLIOGRÁFICAS}

1. Figueiredo LTM, Fonseca BAL. Dengue. In: Veronesi R, Focaccia R. Tratado de Infectologia, Editora Atheneu. 2ª Edição, Rio de Janeiro. p. 204-217, 2002.

2. Fundação Nacional de Saúde. Ministério da Saúde. Programa Nacional de Controle da Dengue. http://www.funasa.gov.br/epi/dengue/dengue0.htm, 2002.

3. Guzmán MG, Kourí G. Dengue: an update. The Lancet Infectious Diseases 2: 33-42, 2002.

4. Harris E, Videa E, Perez L, Sandoval E, Tellez Y, Perez ML, Cuadra R, Rocha J, Idiaquez W, Alonso RE, Delgado MA, Campo LA, Acevedo F, Gonzalez A, Amador JJ, Balmaseda A. Clinical, epidemiologic, and virologic features of dengue in the 1998 epidemic in Nicaragua. American Journal of Tropical Medicine and Hygiene 63: 5-11, 2000.

5. Massad E, Coutinho FA, Burattini MN, Lopez LF. The risk of yellow fever in a dengue-infested area. Transaction of Royal Society of Tropical Medicine and Hygiene 95: 370-374, 2001.

6. Miagostovich MP, Santos FB, Simone TS, Costa EV, Filippis AMB, Schatzmayr HG, Nogueira RMR. Genetic characterization of dengue virus type 3 isolates in the States of Rio de Janeiro, 2001. Brazilian Journal of Medical and Biological Research 35: 869-872, 2002.

7. Nogueira RMR, Miagostovich MP, Filippis AMB, Pereira MAS, Schatzmary HG. Dengue Virus Type 3 in Rio de Janeiro, Brazil. Memórias do Instituto Oswaldo Cruz 96: 925-926, 2001.

8. Nogueira RMR, Miagostovich MP, Schatzmary HG, Santos FB, Araújo ESM, Filippis AMB, Souza RV, Zagne SMO, Nicolai C, Baran M, Texeira Filho G. Dengue in the State of Rio de Janeiro, Brazil, 1986-1998. Memórias do Instituto Oswaldo Cruz 94: 297-304,1999.

9. Schatzmayr HG. Dengue Situation in Brazil by Year 2000. Memórias do Instituto Oswaldo Cruz 95: 179-181, 2000.

10. Tauil PL. Urbanização e Ecologia do Dengue. Caderno de Saúde Pública 17: 99-102, 2001.

11. Tauil PL. Aspectos Críticos do Controle do Dengue no Brasil. Caderno de Saúde Pública 18: 867-871, 2002.

12. Werneck GL, Almeida LM. Validade em estudos epidemiológicos. In: Medronho RA, Carvalho DM, Bloch KV, Luiz RR, Werneck GL (eds) Epidemiologia, Editora Atheneu, p.199-212, 2002. 